\title{
Comparing of K-Means, K-Medodis and Fuzzy C- means Cluster Method for Analog Modulation Recognition
}

\author{
Y. KAYA, D. AVCI and M. GEDİKPINAR
}

\begin{abstract}
A modulation process is required to transmit analog signals with higher quality. Modulation is the process of transporting the signal by another carrier signal. This study aims to process analog signals. Using 200 samples of each of the six types of analog modulation modules. Nowadays these are Amplitude Modulation (AM), Double Side Band (DSB), Upper Side Band (USB), Lower Side Band (LSB), Frequency Modulation (FM) and Phase Modulation(PM) respectively. In the study an intelligent clustering method has been developed. The 5th level Discrete Wavelet Transform (DWT), Norm Entropy and Energy properties of AM, DSB, USB, LSB, FM and PM analog modulated signals have been removed during feature extraction phase. The results have been compared using K-Means, K-medoid and Fuzzy Cmeans (FCM) algorithms using a feature vector of $6 \times 2 \times 1200$ obtained at the feature extraction stage and carrying out smart intelligent clustering for recognition. The most successful result has been obtained with FCM of $85.75 \%$.
\end{abstract}

Index Terms -Analog modulation, wavelet transform, entropy, energy, K-Means, K-medoid, Fuzzy C-means

\section{INTRODUCTION}

$\mathrm{T}$ HE INFORMATION to be used in communication is not used where it is produced. Information may need to be moved to different locations. This distance may also near or far distances. The information / signals produced are produced as low frequency. Low-frequency information is difficult to transport. Therefore, it uses high frequencies to carry this signal. The process of transmitting a low frequency.

YUSUF KAYA, is with Department of Institute of Science and Technology Firat University,Elazıg, Turkey,(e-mail: yucufkaya23@gmail.com).

(D) https://orcid.org/0000-0003-2404-1678

DERYA AVCI,is with Department of Computer Technology Firat University,Elazıg, Turkey, (e-mail: davci@firat.edu ).

(iD https://orcid.org/0000-0002-5204-0501

MEHMET GEDIKPINAR, is with Department of Electrical and Electronics Engineering Firat University,Elazıg, Turkey, (e-mail: mgedikpinar@firat.edu ).

(iD https://orcid.org/0000-0002-1045-7384

Manuscript received May 14, 2019; accepted June 6, 2019.

DOI: $10.17694 /$ bajece. 564960
Low-frequency information is difficult to transport. Therefore, it uses high frequencies to carry this signal. The process of transmitting a low frequency information from one place to another with a high frequency signal is called modulation. Information signal is moved by modulation of amplitude, phase and frequency of carrier signal by modulation [1]. The aim of this study is to select the appropriate method for the healthy transport and processing of analog signals. Also, using real human audio signals, a carrier-clustered intelligent clustering method has been developed for recognition using 200 samples from each of 6 kinds of analog modulation types. K-averages, Kmedoid and Fuzzy C Averages (FCM) algorithms for the identification of the carrier with a clustered smart comparison was compared. Some of the previous modulation studies related to this field are given below. Erdem Yakut S. has formed an intelligent system for the purpose of analog modulation recognition. In the developed smart system, Wavelet Transform (WT), Artificial Neural Networks (ANN) have used Adaptive Network Based Fuzzy Inference System[2]. Guldemir, H. et al. have used 5 kinds of modulation (Amplitude Modulation, Double Side Band Modulation, Frequency Modulation, Upper Single Band Modulation, Lower Single Band Modulation) for clustering process. They compared performance using K-means, Fuzzy C-means, Mountain and Subtractive clustering methods [3]. Avc1, E., made a strong feature in order to classify the targets created by the radar correctly. He used pattern recognition methods to accomplish this purpose. These analyzes were evaluated by integrated feature extraction methods [4]. Sengür, A. et al. have performed the modulation recognition process using Bayes decision rule method to classify analog modulated communication signals [5]. Fidan S. modeled electromagnetic waves emitted in a waveguide by wavelet transform. In study, he placed a wavelet transform filter and a reverse wavelet transform filter, respectively, at the beginning and the end of a waveguide. Thus, it has been shown that solutions can be produced with the desired accuracy by comparing the values obtained for the wavelet types [6]. Demren, E., aimed at the processing of signals with sudden changes in signals and static components. In order to achieve this, he examined a signal processing method to examine high frequency resolution and high time resolution together. In this study, "Wavelet Transformations", another method of frequency-time analysis, has been investigated. In this study, the applicability of automotive, noise and vibration signals has been studied through examples [7]. Işık, M., has compared 
BALKAN JOURNAL OF ELECTRICAL \& COMPUTER ENGINEERING， Vol. 7, No. 3, July 2019

the performance of algorithms by performing various tests on synthetic and real data sets by using partitioned clustering methods [8].

\section{MATERIAL AND METHOD}

In this study, using 200 samples from each of 6 types of analog modulation using human audio signals, a carrier-specific intelligent clustering method has been developed for recognition. The study consists of two stages as feature extraction and clustering. In the feature subtraction phase of the study, distinguishing features of the study by using Level 5 Discrete Wavelet Transform (DWT), energy and entropy methods have been determined. Using the feature vector obtained in the feature subtraction stage, K-averages, K-medoid and Fuzzy C Averages (FCM) algorithms have been used to perform intelligent clustering.

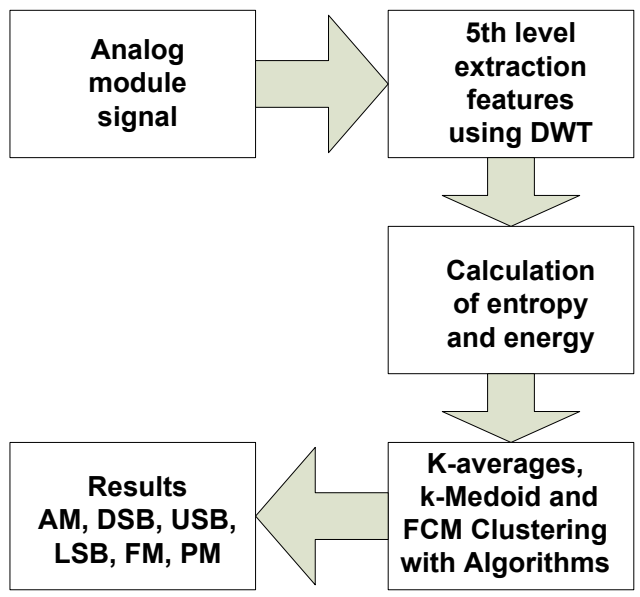

Fig. 1. Flow Diagram of the System Used

\section{A. Discrete Wavelet Transform (DWT)}

Discrete Wavelet Transform (DWT) is used to better analyze markers. DWT uses a narrower windowing dimension in the wider and low-frequency sections in high-frequency sections [6]. Distinguishes DWT from other conventional modulation recognition methods is the use of different windowing techniques [9]. Thus, the windows to be created for the transmission of the signal, where the frequency information, the window wide and also where the time zone is important window areas may be preferred. In this way, the time information of the transmitted signals is also visible. DWTs allow these nonstationary signals to be carried [10]. DWTs are used in applications such as astronomy, fingerprint verification, geophysics, internet traffic regulation, meteorology, computer graphics analysis, speech recognition [11]. With ADD, two signs called Approximate (A) and Details (D) are created. A sign is composed of wide time - low frequency and B is narrow time high frequency. Figure 2 shows a simple block diagram of DWT.

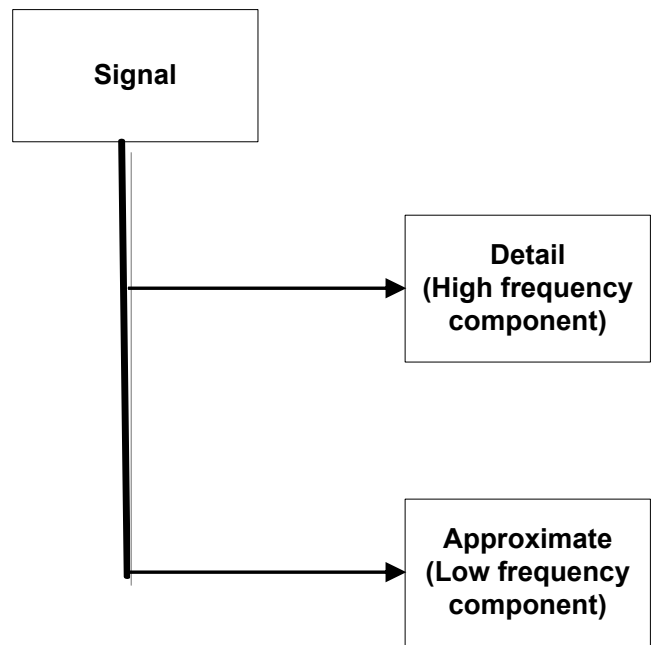

Fig. 2. DWT Simple Block Diagram

\section{B. Entropy}

The concept of entropy was first used by American scientist Claude Shannon in 1948 as part of the theory of probability and knowledge [12]. Entropy can be defined as randomness, irregularity or uncertainty in a system, if it is expressed in a comprehensive manner. Different entropy calculation techniques are used in signal processing field. Some of those; Shannon, Norm, Threshold and Logarithmic Energy methods. In the signal processing, if the sign is stationary, it has a low spectrum with a low spectrum in the frequency region. If the opposite sign is not stationary, the frequency spectrum is spread over a wide band and causes a large entropy value [13].

\section{K-Means}

$\mathrm{K}$-means (KM), one of the first clustering methods, was proposed by Hugo Steinhaus in 1957. In 1967, this method was developed by J.B. by MacQueen [14].

It is defined as one of the learning methods without training. Once clustering with KM, objects most similar to each other are placed in the same set around a center. This placement is based on the distance of the objects to each other. Since each object will be in a group after $\mathrm{KM}$, there is a noticeable difference between the groups. The determination of $\mathrm{k}$ points by a sample $\mathrm{k}$-means clustering method and the clustering steps of the units are shown in Figure 3 [15],[16].

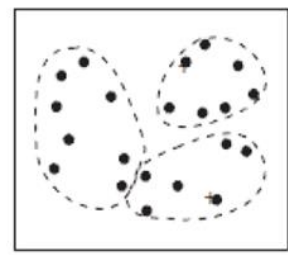

a) initial clustering

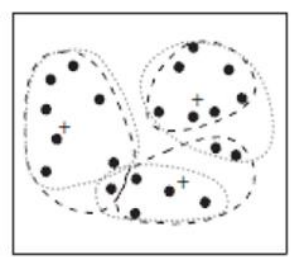

b) iteration

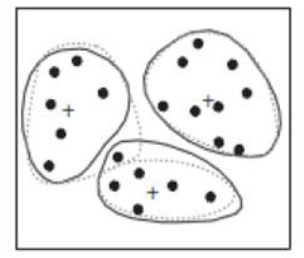

c) final clustering

Fig. 3. Random Clustering of $\mathrm{K}$ 
BALKAN JOURNAL OF ELECTRICAL \& COMPUTER ENGINEERING, Vol. 7, No. 3, July 2019

\section{K-medoids}

With the s, the medoid is taken instead of the midpoint of the cluster. Medoid refers to the data in the center of the cluster to be created. K-medoids consist of $\mathrm{k}$-set and $\mathrm{n}$-data. The number of $\mathrm{k}$ sets is determined by the user. According to the data at the specified k-points, the other n-data are assigned to clusters that are close to them [17]. The cluster center and the midpoint can vary considerably with new data to be included in the central point of clustering with KM. However, if large data is included in the system, the processes may be extended in this method. A clustering with K-medoids is as shown in Figure 4. The cluster is used as one of the central data [18].

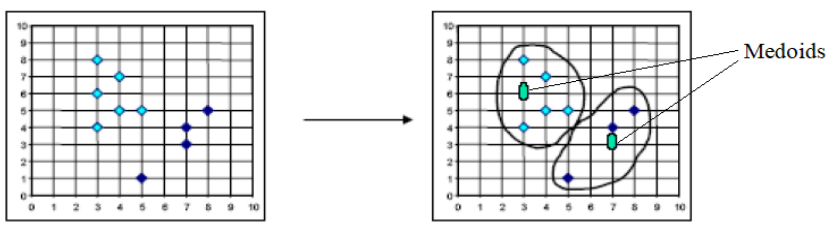

Fig. 4. Clustering with K-medoids

\section{E. Fuzzy C-meanss}

FCM was first proposed by Dunn in 1973. Performs clustering using fuzzy logic [19]. In classical clustering methods, each data must belong to a set and the data must be expressed as data "0" or " 1 ". However, FCM transactions can also take any value that includes "0-1" range. When clustering with FCM, each data does not have to belong to a cluster. The sum of the value of each data belonging to all clusters should be "1" [20]. A clustering with classical clustering and comparison of the clustering with FCM is given in Figure 5. In Figure 5, H1 and $\mathrm{H} 2$ are classical clustering, $\mathrm{F} 1$ and $\mathrm{F} 2$ are clustering with FCM [21].

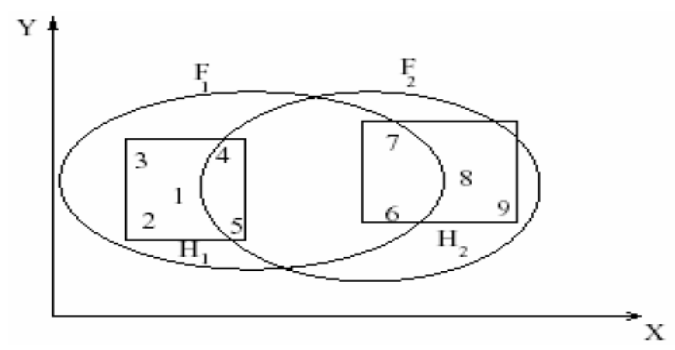

Fig. 5. Clustering with Classic Clustering and FCM

When the FCM is clustered with the FCM algorithm, the first data value is randomly assigned. Using these values, the location of the cluster center is determined with the help of iterative updates. FCM assigns the data to the group that is close to "1"according to the membership degree. FCM has advantages such as facilitates recognition of ambiguous situations, high ability to find overlapping clusters, a flexible structure, and little impact on noise [22].

\section{APPLICATION}

The actual audio signal is used as an information signal for use in the application. Generally, 6 types of analog modulation are used, including Amplitude Modulation (AM), Double Side Tape (DSB), Upper Side Band (USB), Lower Side Tape (LSB), Frequency Modulation (FM), Phase Modulation (PM). With the aim of recognizing by using 200 samples from each of the said modulation types, carrier-based intelligent clustering method has been developed. In order to obtain the signals with analog modulated, Gaussian white noise has been added to the signal between 0 - $70 \mathrm{~dB} \mathrm{~S}$ (Signal) / N (Noise) and 20 signals have been generated from each modulation type. The starting angle and modulation index $(\mathrm{m})$ of the 20 signals created by adding the noise have been changed to 200 modal signs for each modulation type. DWT uses a narrower windowing dimension in the high-frequency sections and in the larger low-frequency sections to better analyze the signals. In the wavelet transform, there are two types of signs: Detail (D) and Approximate (A). In this study, the additive obtained from the 5th level decomposition of the wavelet transform is used. The 5-level decomposition of DWT has been used for feature extraction because the best value of the signal has been found to be Level 5 according to Entropy measurement. Since 5 level decomposition of DWT is taken, 6 signatures have been formed including 5 "D" and 1 "A" sign. Norm entropy and Energy values of each of these signals have been calculated and the feature has been removed for each signal. 6 (the number of DWT features of each modulated signal) X 2 (Norm entropy and number of energy properties of each modulated signal) X 6 (modulation type) x 200 (number of samples used for each modulation type) dimension vector has been obtained. By using the obtained feature vector, it has been carried out intelligent clustering with carrier to recognize by KM, K-medoid and FCM Algorithms. MATLAB 2018 software has been used in this study [23]. The pseudo-code representation of the processing steps used in the application is as follows:

Step 1: To make the Analog signals to be decomposed to create a more complex structure by adding noise in the range of 0-70 $\mathrm{db}$ to the signal to be separated,

Step 2: To increase the data set, replicate the signals by changing the initial angle and modulation index $(\mathrm{m})$ of the signal,

Step 3: To feature extraction the 5-level decomposition of DWT and energy-entropy values to increase the number of attributes, Step 4: Performing Clustering with the Removed feature vector.

\section{A. Distinctive Features Extraction}

In this study, in the 5th level division of the DWT, there are 5 Detail (D), 1 Approximate (A). 6 signals have been created. Norm entropy and Energy values of each of these signals have been calculated and the property has been omitted for each sign. 6 (the number of DWT features of each modulated signal) X 2 (Norm entropy and number of energy properties of each modulated signal) X 6 (modulation type) x 200 (number of 
BALKAN JOURNAL OF ELECTRICAL \& COMPUTER ENGINEERING， Vol. 7, No. 3, July 2019

samples used for each modulation type) dimension vector has been obtained. 5 Detail (D) and 1 Approximate (A) signals have been obtained from each of the modulation signals used. Figure 6 shows the structure of the 5 level wavelet transform.

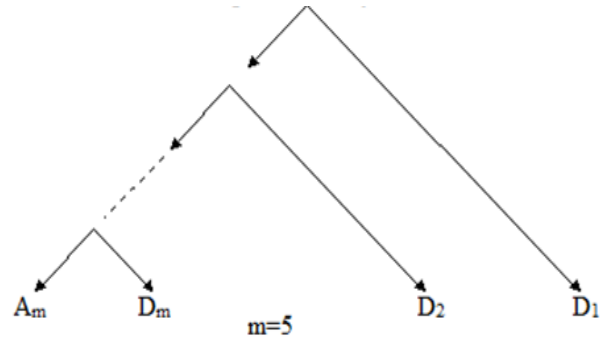

Fig. 6. Structure of 5th Level Wavelet Transform

\section{B. Recognition of Modulation Type by Clustering Methods}

Using the $6 \times 2 \times 1200$ feature vector obtained in the feature subtraction phase, intelligent clustering with carrier has been performed for recognition by K-means, K-medoids and FCM algorithms. For the K-means clustering method in Table 1, for the K-medoids clustering method in Table 2, for the FCM

clustering method in Table 3 show the confusion matrix The values in the row and column in the same row in the confusion matrix indicate that the clustering is done correctly, and that the different queues indicate clustering. For example, in Table 1, the intersection value of $\mathrm{AM}$ in the row with $\mathrm{AM}$ and 174 of the AM modulation were correct, AM was clustered as AM. AM states that 6, $6 \mathrm{AM}$ at the intersection of the DSB are incorrectly clustered as the DSB. AM states that 5, AM at the intersection of the USB are incorrectly clustered as the USB. AM states that 7, $7 \mathrm{AM}$ at the intersection of the LSB are incorrectly clustered as the LSB. AM states that 3, $3 \mathrm{AM}$ at the intersection of the FM are incorrectly clustered as the FM. Since 5 PM states that are incorrectly clustered.

TABLE I

K-MEANS CONFUSION MATRIX FOR CLUSTERING METHOD

\begin{tabular}{|c|c|c|c|c|c|c|c|}
\hline Modulation Type & $\mathbf{A M}$ & DSB & USB & LSB & FM & PM & Total Success \\
\hline AM & 174 & 6 & 5 & 7 & 3 & 5 & $\% 87$ \\
\hline USB & 7 & 5 & 173 & 3 & 5 & 7 & $\% 86.5$ \\
\hline FM & 2 & 6 & 4 & 3 & 170 & 5 & $\% 85$ \\
\hline PM & 3 & 5 & 6 & 4 & 4 & 168 & $\% 84$ \\
\hline \multicolumn{7}{|c|}{ Average Performance } & $\% 84.916$ \\
\hline
\end{tabular}

TABLE II

K-MEDOIDS CONFUSION MATRIX FOR CLUSTERING METHOD

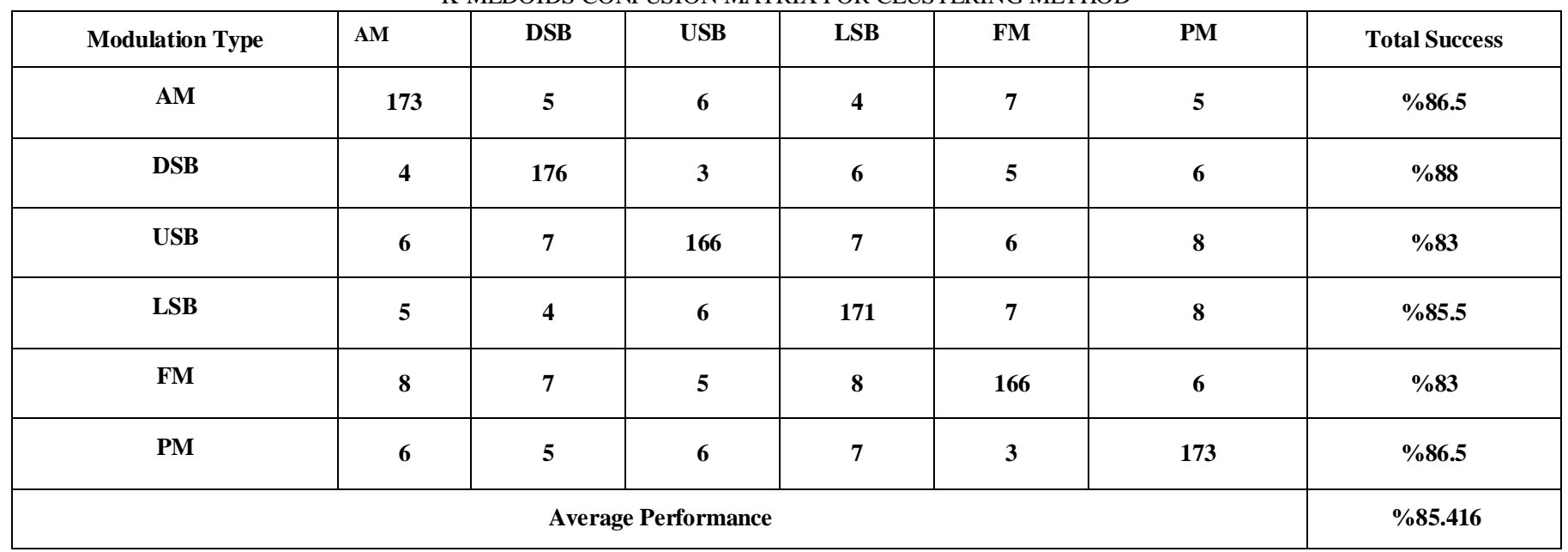


BALKAN JOURNAL OF ELECTRICAL \& COMPUTER ENGINEERING, Vol. 7, No. 3, July 2019

TABLE III

FCM CONFUSION MATRIX FOR CLUSTERING METHOD

\begin{tabular}{|c|c|c|c|c|c|c|c|}
\hline Modulation Type & $\mathbf{A M}$ & DSB & USB & LSB & FM & PM & Total Success \\
\hline $\mathbf{A M}$ & 175 & 4 & 6 & 7 & 3 & 5 & $\% 87.5$ \\
\hline DSB & 8 & 167 & 7 & 6 & 7 & 5 & $\% 83.5$ \\
\hline USB & 5 & 7 & 169 & 6 & 7 & 6 & $\% 84.5$ \\
\hline LSB & 4 & 5 & 6 & 173 & 6 & 7 & $\% 86.5$ \\
\hline FM & 5 & 3 & 6 & 5 & 175 & 6 & $\% 87.5$ \\
\hline PM & 4 & 3 & 6 & 3 & 4 & 170 & $\% 85$ \\
\hline \multicolumn{7}{|c|}{ Average Performance } & $\% 85.75$ \\
\hline
\end{tabular}

\section{RESULT CONCLUSION}

Carrier intelligent clustering method is developed for recognition using 200 samples from each of 6 kinds of analog modulation types including AM, DSB, USB, LSB, FM, PM and $6 \times 200$ samples have been used in this method. The feature vector of $6 \times 2 \times 1200$ has been used in the feature extraction stage. Table 1, Table 2 and Table 3 have been formed with the obtained data. The results obtained from the tables have been

\section{REFERENCE}

[1] https://slideplayer.biz.tr/slide/2683446 [Access: 23-Sep-2018]

[2] S. Erdem Yakut. "An intelligent classification system based on wavelet transform in analog modulations." M.Sc. Thesis, F.Ü. Graduate School of Natural and Applied Sciences, Elazığ, 2007.

[3] H. Guldemır, A. Sengur. "Comparison of clustering algorithms for analog modulation classification." Expert Systems with Applications, 30(4), 642649K. Elissa, 2006.

[4] E. Avc1. "Intelligent radar target recognition system." Ph.D. Thesis, F.Ü. Graduate School of Natural and Applied Sciences, Elazı ğ, 2005.

[5] A.Şengür, İ.Türkoğlu. "Classifying Analogue Modulated Communication Signals Using Bayes Decision Criterion." Sakarya University Journal of Science, Vol.7, No.3, 2003, pp. 32-36.

[6] S. Fidan. "Modeling of the electromagnetic wave emitted in the waveguide by wavelet transformation." M.Sc. Institute of Science and Technology, Ankara, 2006.

[7] E.Demren. "Comparison of wavelet transform with fourier transform and its application.” MS Thesis, İ.T.Ü. Institute of Science and Technology, Istanbul, 2015.

[8] M. Işık. "Data mining applications with partitioned clustering methods." M.Sc. Institute of Science and Technology, Istanbul, 2016.

[9] H.Polat, M.Akin, and M.S.Özerdem. "The comparison of wavelet and empirical mode decomposition method in prediction of sleep stages from EEG signals." In Artificial Intelligence and Data Processing Symposium (IDAP), 2017 International (pp. 1-5). IEEE.

[10] E.Avcı, İ.Türkoğlu and M.Poyraz. "Intelligent Target Recognition Based on Wavelet Adaptive Network Based Fuzzy Inference System." Lecture Notes in Computer Science, Springer-Verlag, 3522, 594-601, 2005.

[11] Ö. Arslan. "Investigating the most appropriate main wavelet function for Turkish phonemes." M.Sc. Institute of Science and Technology, İzmir.Gray, R. M., Entropy and information. In Entropy and Information Theory, Publisher: Springer, (pp. 21-55) New York, ISBN-13: 9781441979698, 2014 analyzed and $84.916 \%$ for the K-means clustering method, $85.416 \%$ for the K-medoids clustering method and $85.75 \%$ for the FCM clustering method. As a result, $85.75 \%$ of the best performance has been found to belong to the FCM clustering method. Afterwards, different modulation types and different clustering algorithms can be used in the studies to be carried out.

[12] R.R. Coifman, M.V. Wickerhauser. "Entropy based algorithms for best basis selection." IEEE Transaction on Information Theory, 38, 2, 713-718, 1992].

[13] A.Çokgüngördü. "Base stations with the help of clustering method, using the assisted site." M.Sc. Institute of Science and Technology, Istanbul,2017.

[14] S.Atal. "Fuzzy clustering analysis and clustering of OECD countries in terms of development." Master Thesis, O.G.Ü. Graduate School of Natural and Applied Sciences, Eskişehir, 2015.

[15] I. Ş. I. K..Meltem, A.Y. Çamurcu. "K-Means, K-Medoids Ve Bulanık CMeans Algoritmalarının Uygulamalı Olarak Performanslarının Tespiti." İstanbul Ticaret Üniversitesi Fen Bilimleri Dergisi, Vol.6, No11, 2007, pp.31-45.

[16] B. Çağlar. "Evaluation of spatial data by clustering analysis." Master Thesis, N.E.Ü. Institute of Science and Technology, Konya, 2018.

[17] S. Kırmızıgül Çalışkan. "K.KNN: detection of penetration in networks by clustering and closest neighboring method." Master Thesis, G.Y.T.E. Institute of Engineering and Science, Gebze, 2008.

[18] O.Taşova. "Face recognition with artificial neural networks." MS Thesis, D.E.Ü. Institute of Science and Technology, İzmir, 2011.

[19] K.Eset. "Investigation of segmentation methods in lung pet images" M.Sc. Institute of Science and Technology, Kayseri, 2016.

[20] A.T. Alper. "Analog communication, Lecture Notes." M.Ü. Faculty of Engineering, Mersin, 2010.

[21] A. Saygili, "S. Albayrak. Meniscus segmentation and tear detection in the knee MR images by fuzzy c-means method." In 2017 IEEE International Conference on Signal Processing and Communications Applications Conference (SIU), IEEE, 2017. https://doi.org/10.1109/SIU.2017.7960183

[22] Aydemie,E. "Weka ile Yapay Zeka”,Secgin Yayınevi, 2018

[23] MATLAB Company, 2018 


\section{BIOGRAPHIES}

Yusuf $\boldsymbol{K A} \boldsymbol{A} \boldsymbol{A}$ - He was born in 1974 in Elazı $\breve{g}$.

He completed his primary, secondary and high school education in Elazı $\breve{g}$. In 1992, he graduated from Marmara University Faculty of Technical Education, Department of Electrical Education and graduated in 1996. He was appointed as a teacher of electricity to Afyonkarahisar by the Ministry of National Education. He is still performing this duty at Gazi Vocational and Technical Anatolian High School in Elazığ. He is master student in Firat University.

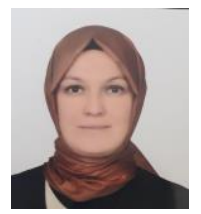

Derya A VCI-She was born in Malatya Turkey. She received a master's degree from Firat University Electronic and Computer Education. She received Ph.D. degree from Firat University Electrical and Electronic Engineering. Her interesting areas are pattern recognition, image processing, intelligent systems, artificial intelligence systems.

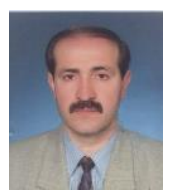

Mehmet GEDIKPINAR- He received the BS degree from the Department of Electrical Education, Gazi University, the MSc degree from Institute of Science and Technology, Firat University,the PhD degree from Institute of Science and Technology, Gazi University respectively. His research interests include intelligent control, electrical machines and designing, electronic instruments and measurements, analyzing of circuit. 